\title{
Identification of Aedes aegypti cis-regulatory elements that promote gene expression in olfactory receptor neurons of distantly related dipteran insects
}

Keshava Mysore ${ }^{1,2}$, Ping $\mathrm{Li}^{1,2}$ and Molly Duman-Scheel ${ }^{1,2,3^{*}}$ (D)

\begin{abstract}
Background: Sophisticated tools for manipulation of gene expression in select neurons, including neurons that regulate sexually dimorphic behaviors, are increasingly available for analysis of genetic model organisms. However, we lack comparable genetic tools for analysis of non-model organisms, including Aedes aegypti, a vector mosquito which displays sexually dimorphic behaviors that contribute to pathogen transmission. Formaldehyde-assisted isolation of regulatory elements followed by sequencing (FAIRE-seq) recently facilitated genome-wide discovery of putative $A$. aegypti cis-regulatory elements (CREs), many of which could be used to manipulate gene expression in mosquito neurons and other tissues. The goal of this investigation was to identify FAIRE DNA elements that promote gene expression in the olfactory system, a tissue of vector importance.

Results: Eight A. aegypti CREs that promote gene expression in antennal olfactory receptor neurons (ORNs) were identified in a Drosophila melanogaster transgenic reporter screen. Four CREs identified in the screen were cloned upstream of GAL4 in a transgenic construct that is compatible with transformation of a variety of insect species. These constructs, which contained FAIRE DNA elements associated with the A. aegypti odorant coreceptor (orco), odorant receptor 1 (Or1), odorant receptor 8 (Or8) and fruitless (fru) genes, were used for transformation of A. aegypti. Six A. aegypti strains, including strains displaying transgene expression in all ORNs, subsets of these neurons, or in a sexspecific fashion, were isolated. The CREs drove transgene expression in A. aegypti that corresponded to endogenous gene expression patterns of the orco, Or1, Or8 and fru genes in the mosquito antenna. CRE activity in A. aegypti was found to be comparable to that observed in $D$. melanogaster reporter assays.
\end{abstract}

Conclusions: These results provide further evidence that FAIRE-seq, which can be paired with D. melanogaster reporter screening to test FAIRE DNA element activity in select tissues, is a useful method for identification of mosquito cis-regulatory elements. These findings expand the genetic toolkit available for the study of Aedes neurobiology. Moreover, given that the CREs drive comparable olfactory neural expression in both A. aegypti and D. melanogaster, it is likely that they may function similarly in multiple dipteran insects, including other disease vector mosquito species.

Keywords: Mosquito, Sensory, Antenna, Neuron, Enhancer, FAIRE, Aedes aegypti, Drosophila melanogaster, Dengue, Zika

\footnotetext{
* Correspondence: mscheel@nd.edu

${ }^{1}$ Department of Medical and Molecular Genetics, Indiana University School of

Medicine, 1234 Notre Dame Avenue, Raclin-Carmichael Hall, South Bend, IN

46617, USA

${ }^{2}$ The University of Notre Dame Eck Institute for Global Health, Notre Dame,

IN 46556, USA

Full list of author information is available at the end of the article
}

(c) The Author(s). 2018 Open Access This article is distributed under the terms of the Creative Commons Attribution 4.0 International License (http://creativecommons.org/licenses/by/4.0/), which permits unrestricted use, distribution, and

reproduction in any medium, provided you give appropriate credit to the original author(s) and the source, provide a link to the Creative Commons license, and indicate if changes were made. The Creative Commons Public Domain Dedication waiver (http://creativecommons.org/publicdomain/zero/1.0/) applies to the data made available in this article, unless otherwise stated. 


\section{Background}

Mosquito control is the primary mechanism for preventing dengue, yellow fever, chikungunya and Zika, all of which result from viruses transmitted by the daytime-biting mosquito Aedes aegypti, which is closely associated with humans and their urban dwellings. The emergence of insecticide resistance and a lack of support for mosquito control programs compromise current strategies for managing mosquitoes [1]. It is therefore critical that we identify new tools to both study mosquito biology and combat these insect vectors of human disease-causing pathogens. Mosquito behaviors such as blood meal acquisition, courtship and oviposition have attracted the attention of biologists for years. Knowledge of the neurogenetic basis for these and other behaviors would facilitate modification of the behaviors for vector and disease control. Unfortunately, analysis of the neurogenetic basis for insect behavior has largely been restricted to Drosophila melanogaster, a genetically-tractable dipteran insect and genetic model organism [2]. While hundreds of neuron-specific GAL4 driver lines enable sophisticated perturbation of the fruit fly nervous system [3, 4], we lack comparable genetic tools for analysis of the neurophysiological basis of behaviors in most insects, including mosquitoes.

Likewise, knowledge concerning the extent of sexual dimorphisms in the structure of the central and sensory nervous systems, the regulation of sex-specific behaviors by sexually dimorphic neurons, as well as the developmental genetic basis for sexually dimorphic behaviors is limited in all organisms, even D. melanogaster, but especially in non-model organisms, including mosquitoes [2, 5]. Advancements in the methodology for studying mosquito neurogenetics would help to elucidate the genes that regulate mosquito sexual dimorphism, including the development and function of neural circuitries that promote host-seeking, blood meal acquisition and feeding behavior, mating and oviposition, all of which may represent genetic targets for vector control [2]. Studies in $D$. melanogaster have begun to reveal the genetic mechanisms that underlie sexually dimorphic behavior in insects. For example, sex-specific splicing of the D. melanogaster fruitless (fru) gene, which encodes a transcription factor, results in male- and female-specific splice forms that contribute to sexually dimorphic behaviors such as courtship and aggression in flies [6-9]. Recent studies in the olfactory system have demonstrated that the actual expression of fru is also sexually dimorphic, and that fru expression serves as a molecular marker for neurons participating in sex-specific behaviors [10]. The detection of sex-specific splice forms of $A$. aegypti fru suggests that it functions as a modulator of sexually dimorphic behavior in $A$. aegypti [11], but the function of this gene has not yet been directly assessed in mosquitoes.
Although the GAL4-UAS binary system for manipulation of gene expression in D. melanogaster neurons has been introduced in $A$. aegypti [12], very few GAL4 lines are currently available. This is largely due to the lack of known CREs in mosquitoes. FAIRE-seq, has emerged as a powerful high-throughput tool for global CRE discovery [13]. FAIRE results in the preferential recovery of open chromatin DNA fragments that are not bound by nucleosomes, an evolutionarily conserved indicator of regulatory activity, which are then sequenced through next-generation sequencing [13-16]. We recently utilized FAIRE-seq to profile open chromatin and identify regulatory elements throughout the genome of $A$. aegypti [17]. The results of this investigation [17] provided evidence that FAIRE-seq is a powerful tool for identification of regulatory DNA in the mosquito genome. We are therefore mining the FAIRE-seq data set for regulatory elements that function in tissues of vector importance.

Here, we describe the identification and characterization of CREs that drive gene expression in the olfactory system, a sensory system that is critical for many sexually dimorphic mosquito behaviors related to mosquito reproduction and pathogen transmission [2]. The first phase of the study exploits the genetic tractability of $D$. melanogaster, in which transgenic generation is quick, straightforward, and economical. Drosophila reporter assays permitted analysis of $A$. aegypti FAIRE DNA elements of interest, leading to identification of CREs that promote gene expression in antennal olfactory receptor neurons (ORNs). Characterization of the Drosophila reporter lines facilitated down-selection of four elements for the direct transformation of $A$. aegypti. CREs that promote gene expression in all $A$. aegypti antennal ORNs, subsets of these neurons, as well as in a sex-specific manner, were identified. The results of this study demonstrate that the regulatory elements function comparably in two distantly related insects, suggesting that they might be used for modification of gene expression, including sex-specific gene expression, in the olfactory systems of $A$. aegypti as well as additional mosquito species and other dipteran insects. These tools, particularly the sex-specific gene driver, may promote the elucidation of new methods for control of disease vector mosquitoes.

\section{Methods \\ Mosquito rearing}

Mosquitoes were reared as previously described [18]. A membrane blood-feeding system was employed in conjunction with commercially supplied sheep blood (Hemostat Laboratories, Dixon, CA). Following establishment of each transgenic strain, an eye-specific genetic marker was selected in subsequent generations for continued maintenance of the strain. Egg libraries are also being maintained for the transgenic strains. 


\section{Drosophila melanogaster transgenic reporter generation and analysis}

Transgenic constructs were prepared as described in Behura et al. [17]. In summary, FAIRE DNA elements of interest (Table 1) were PCR-amplified from A. aegypti genomic DNA and cloned into plasmid pattBnucGFPs (graciously provided by $\mathrm{M}$. Halfon), a phiC31-enabled Drosophila transformation vector containing EGFP under the control of a minimal $h s p 70$ promoter. Transgenic Drosophila were produced at Rainbow Transgenic Flies, Inc. (Camarillo, CA) by injection into line $P B a$ $c\{y[+]$-attP-9A\}VK00027 (Bloomington Stock Center \#RRID:BDSC_9744 [19]). In each of two replicate experiments, tissue from $10 w+$ male and $10 w+$ female transgenic animals was collected and fixed as described previously [20]. In total, 80 antennae from each line were evaluated.

\section{Aedes aegypti transgenic construct generation}

A derivative of the PB-GAL4 ECFP construct described in O'Brochta et al. [21] was generously provided by D. O'Brochta. The construct, which is compatible with both $p B a c$ and phiC31 transgenesis, is marked with ECFP driven by a universal insect $3 x P 3$ eye promoter [22]. This construct was modified through insertion of a new multiple cloning site (5'-CGC TAG CGC CGG CAG ATC TCC TAG G-3') between the BgIII and NgoMIV sites downstream of pBacleft and upstream of the open reading frame of GAL4. The addition of this multiple cloning site facilitated insertion of fru, orco, Or1 or Or8 FAIRE regulatory elements (Table 1), which were PCR-amplified, along with an upstream $h s p 70$ minimal promoter, from the pattBnucGFP constructs described above. Constructs were verified through restriction digestion and sequencing.

\section{Aedes aegypti transgenesis and transgenic line characterization}

Aedes aegypti transgenic constructs described above were supplied to the Insect Transformation Facility (ITF) at the University of Maryland College Park facility, which generated the $A$. aegypti transgenics. The facility microinjected 70 A. aegypti (Liverpool strain) eggs (G0 animals) per construct. Following maturation, G0 adults were backcrossed to uninjected individuals. First generation (G1) offspring were reared and screened for ECFP expression. Transgenic G1 individuals were mated to generate transgenic strains. The resulting G2 individuals were screened via PCR with primers specific to the transgene for molecular confirmation of the transgenic line. G2 offspring were then crossed to establish a transgenic line, which was expanded and further characterized. Tissues were fixed and prepared as described [23]. GAL4 expression driven by the FAIRE DNA regulatory element was monitored via in situ hybridization which was performed as described [24] and compared to endogenous gene expression patterns. Riboprobes for these studies were generated as described by Patel [25]; note that the fru riboprobe used in these studies corresponds to sequence that is conserved between the male and female splice forms [11]. For larval studies, in each of two replicate experiments, 40 antennae were dissected from 20 ECFP-positive larvae from each strain; in total 80 antennae were assessed per strain. For adult studies, 60 antennae from 30 ECFP-positive adults of each strain (15 males and 15 males) were assessed.

\section{Imaging and image processing}

Following processing, tissues were mounted in glycerol and imaged on a Zeiss Axioimager equipped with a Spot Flex camera and Spot Digital Imaging software. Confocal imaging was performed at the IUSM Flow Cytometry and Imaging Core facility using a Zeiss 710 confocal microscope and Zen software. Images were analyzed with FIJI ImageJ

Table 1 FAIRE DNA elements assessed in D. melanogaster reporter assays

\begin{tabular}{lll}
\hline FAIRE DNA Element $^{\mathrm{a}}$ & Flanks Gene no. $^{\mathrm{b}}$ & Gene TSS $^{\mathrm{c}}$ \\
\hline supercont1.174:341062-341799 & AAELO05776 (orco ${ }^{\mathrm{C}}$ & supercont1.174:357279 \\
supercont1.1782:38974-39907 & AAEL016970 (Or1) & supercont1.1782:39941 \\
supercont1.671:130269-131236 & AAEL012254 (Or8) & supercont1.671:131254 \\
supercont1.199:700946-702158 & AAEL006301 (fru) & supercont1.199:701824 \\
supercont1.237:1279560-1280173 & AAEL007110 (Or16 ${ }^{\mathrm{c}}$ ) & supercont1.237:1269860 \\
supercont1.160:604315-605761 & AAEL005507 (acj6 ${ }^{\mathrm{c}}$ ) & supercont1.160:435622 \\
supercont1.123:863985-864746 & AAELO04572 (E93) & supercont1.123:868,883 \\
supercont1.54:975577-976601 & AAEL002359 (onecut) & supercont1.54:924002 \\
\hline
\end{tabular}

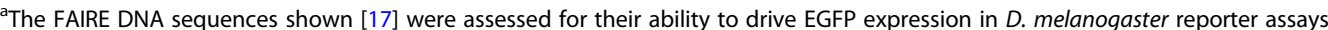

${ }^{\mathrm{b}}$ The flanking genes (gene number and name) and transcription start sites (TSSs) of the flanking genes are noted. Sequences correspond to $A$. aegypti scaffolds reference v.4, which was used in the FAIRE-seq investigation [17]. A subset of these elements (CREs associated with orco, Or1, Or8 and fru) were used for subsequent generation of $A$. aegypti transgenic strains

'Drosophila reporter lines were initially described in Behura et al. [17] and are characterized in further detail in the present investigation 
and Adobe Photoshop CC 2014 software. Signal intensities between the Or1-GAL4a versus $b$ and Or8-GAL4a versus $b$ lines were evaluated through comparison of mean gray values (average signal intensity over the selected area) in each line for the specific cells noted in Fig. $2(n=25)$ and Fig. $3(n=15)$ using Fiji software. The cells selected for these analyses were chosen because they were easily recognized in the antennae of each mosquito assessed. Mean gray value data were statistically analyzed with a paired t-test.

\section{Results}

\section{A screen for A. aegypti antennal CREs in Drosophila}

The A. aegypti FAIRE DNA data set [17] was assessed for regulatory elements residing adjacent to $A$. aegypti odorant receptor $(\mathrm{Or})$ genes, as well as genes encoding transcription factors that function in the A. aegypti antenna to regulate $O r$ gene expression [26]. It was hypothesized that FAIRE DNA elements flanking these genes, which are known to be expressed in Aedes antennal ORNs [26], would regulate gene expression in these neurons. In total, eight FAIRE DNA elements were identified and all were evaluated in this investigation (Table 1). The $A$. aegypti FAIRE DNA sequences were first cloned upstream of enhanced green fluorescent protein (EGFP) in a Drosophila phiC31-enabled transformation vector, and the resulting constructs were used for Drosophila transformation. All eight FAIRE DNA elements (Table 1) were confirmed to drive EGFP reporter expression in D. melanogaster (Fig. 1).

In an effort to select the most promising CREs for subsequent generation of $A$. aegypti transgenics (see below), the Drosophila reporter lines were characterized in detail. First, D. melanogaster reporters generated from FAIRE DNA residing upstream of the A. aegypti E93, onecut and abnormal chemosensory jump (acj6) genes (Table 1) were assessed. These genes encode transcription factors expressed in $A$. aegypti antennal ORNs, in which they are believed to play conserved roles in regulation of the $\mathrm{Or}$ expression code [26]. Antennal expression of EGFP was detected in all three of the Drosophila reporter lines (Fig. 1a-c). In the E93 Drosophila reporter (Fig. 1a), EGFP was expressed broadly in multiple adult ORNs. EGFP expression was restricted to a smaller subset of ORNs in the onecut reporter line (Fig. 1b). EGFP expression in the acj6 Drosophila reporter line, which had previously been documented in the D. melanogaster brain [17], was also detected in a subset of $D$. melanogaster adult antennal ORNs (Fig. 1c).

Drosophila melanogaster reporter lines generated using FAIRE DNA elements located adjacent to A. aegypti Or genes were also assessed (the A. aegypti Or genes were originally described in [27]). Two Drosophila reporter lines preliminarily characterized in the original study [17] had been generated with FAIRE DNA elements flanking the $A$. aegypti orco and Or16 genes. The orco gene encodes the $A$. aegypti odorant coreceptor [28], while Or16 encodes an odorant receptor protein of unknown function [27]. Behura et al. [17] had detected expression of GFP in the adult antennae of both reporter lines; both lines were characterized in further detail here. Analysis of the orco line confirmed that EGFP expression could be detected in all ORNs of the adult antenna (Fig. 1e). Further characterization of the $D$. melanogaster Or16 reporter line confirmed EGFP expression in the fly antenna that was restricted to a subset of these adult ORNs (Fig. 1h). Two additional Drosophila reporter lines, which contained FAIRE DNA upstream of the A. aegypti Or1 (an odorant receptor of unknown function [27]) and Or8 (which encodes a receptor for 1-octen-3-ol, an attractive odor emitted by hosts [29]) genes were generated and assessed. EGFP was also detected in the D. melanogaster adult antennal ORNs of these new reporter lines (Fig. If and g, respectively). EGFP expression was limited to subsets of ORNs in the Or1 and Or8 Drosophila reporter antennae.

A FAIRE DNA element was also identified upstream of $A$. aegypti fru [17]. As discussed above, the fru gene, which is expressed in a sexually dimorphic pattern, is a key regulator of sex-specific behavior in Drosophila [30] and is believed to play comparable roles in $A$. aegypti [11]. Given the lack of tools available to study sexually dimorphic mosquito behaviors [2], this prospective CRE element was also selected for further analysis and used to construct a D. melanogaster EGFP reporter line. Reporter analyses demonstrated that although EGFP was expressed broadly in the adult male $D$. melanogaster antenna (Fig. 1d), it could not be detected in the female antenna (Fig. 1i). These findings suggested that this FAIRE DNA element isolated from $A$. aegypti might be a regulator of sex-specific antennal ORN gene expression.

\section{Generation of $A$. aegypti transgenics}

The next task was to select high-priority CREs for analyses to be performed directly in A. aegypti. For these selections, it was determined that limited CRE activity, spatially and temporally, would be desirable. This could facilitate the specific manipulation of neurons of interest, for example through the expression of toxins for directed neural cell ablation, without concerns for unintended impacts on multiple tissues. Given the importance of sex-specific behaviors to mosquito biology and the transmission of disease-causing pathogens [2], the fru CRE, which did not drive reporter expression in Drosophila embryos (Fig. 1i1), was selected for further analysis in Aedes, in which it was hypothesized that it would promote post-embryonic sex-specific gene expression in the antenna. Regulatory elements upstream of orco, Or1 and Or8 drove specific EGFP expression in Drosophila reporter ORNs (Fig. 1e-g) and not in other reporter tissues (Fig. 1e1, f1, g1), and 

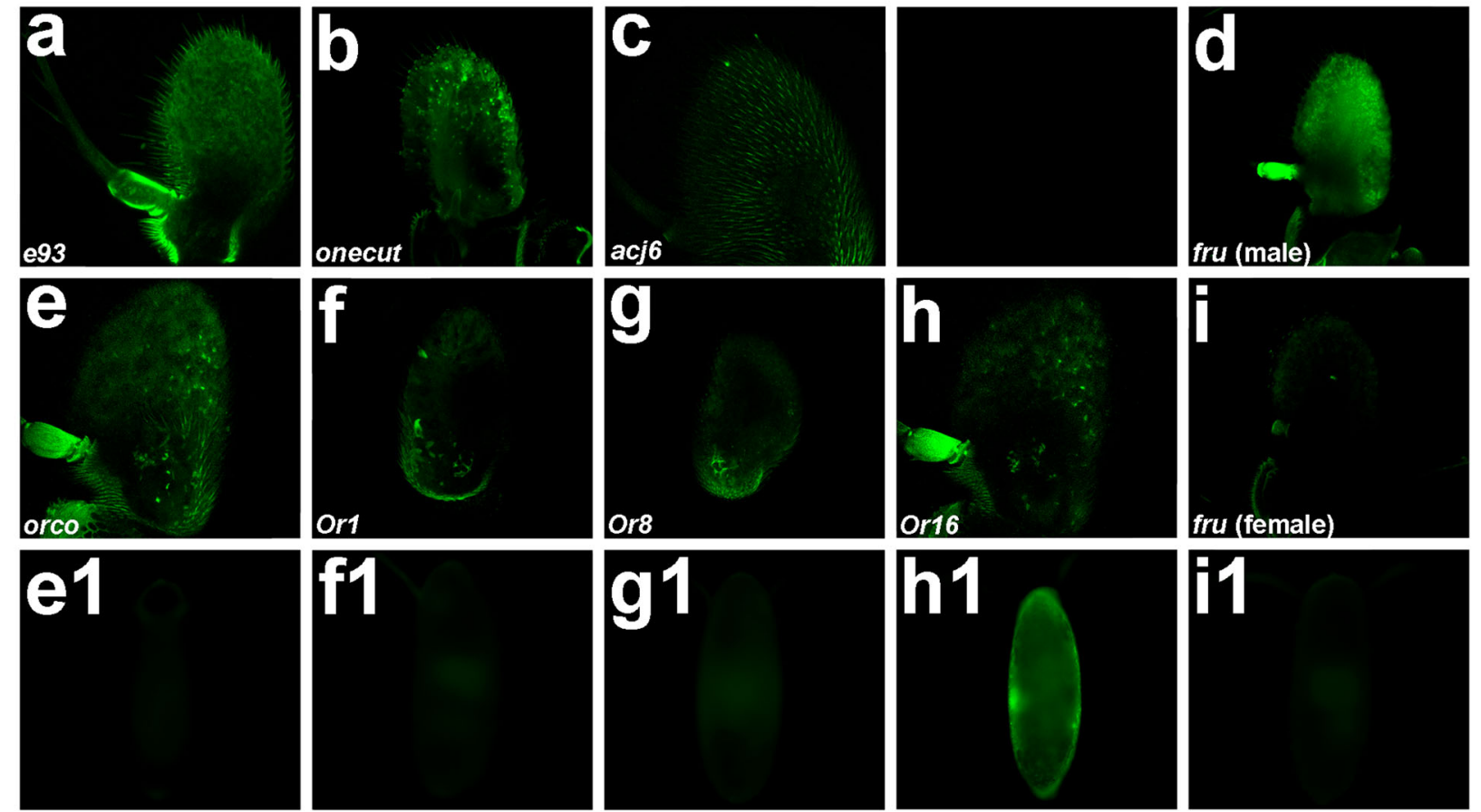

Fig. 1 A D. melanogaster screen identifies A. aegypti CREs that promote gene expression in antennal ORNs. EGFP reporter expression driven by FAIRE DNA sequences flanking the indicated genes (e93 in $\mathbf{a}$, onecut in $\mathbf{b}$, acj6 in $\mathbf{c}$, fru in $\mathbf{d}$, i, and i1, orco in e and $\mathbf{e} 1$, Or 1 in $\mathbf{f}$ and $\mathbf{f} 1$, Or8 in $\mathbf{g}$ and $\mathbf{g 1}$, Or16 in $\mathbf{h}$ and $\mathbf{h} \mathbf{1}$ ) was assessed in a total of 40 female and 40 male adult antennae prepared from replicate experiments. EGFP expression patterns ranged from expression in all (e) or many (a- c) antennal ORNs to very specific subsets of ORNs (f-h). Sex-specific EGFP expression was detected in the adult male antenna (d; compare to female antenna in i). Embryonic expression of EGFP was detected in the Or16 reporter line (h1), but not in the orco (e1), Or1 (f1), Or8 (g1) or fru (i1) Drosophila reporter lines. Proximal is oriented upward in a-i, and anterior is oriented upward in e1-i1

these elements were also selected for Aedes transformations. Based on the Drosophila reporter assays, it was hypothesized that these $A$. aegypti FAIRE elements would drive gene expression in all ORNs (orco), as well as subsets of neurons (Or1 and Or8). The selection of CREs that drive gene expression in both broad as well as narrow subsets of ORNs was intentional, as it is expected to promote flexibility in experimental design in Aedes in the future. The other elements listed in Table 1 were not prioritized for Aedes transgenic generation at this time, as it was predicted that these elements would be active in multiple tissues (Fig. 1h1) [17, 26].

A derivative of the PB-GAL4 ECFP construct described in O'Brochta et al. [21] was used for piggyback ( $p B a c)$-mediated Aedes transformation. The construct, which is marked by ECFP driven by a universal insect $3 x P 3$ eye promoter [22], was modified through insertion of a multiple cloning site (MCS) upstream of the open reading frame of $G A L 4$, which facilitated insertion of the selected FAIRE regulatory elements (Table 1), as well as an $h s p 70$ minimal promoter, upstream of GAL4. GAL4 (like EGFP in the Drosophila assays) served as a reporter in this investigation. One fru-GAL4 strain, an orco-GAL4 strain, two Or1-GAL4 lines and two Or8-GAL4 A. aegypti strains were successfully generated and characterized (Figs. 2 and 3).

Characterization of $A$. aegypti transgenic strains confirms the discovery of regulatory elements that promote gene expression in A. aegypti antennal ORNs

The expression patterns of the GAL4 transgene, which served as a reporter of CRE activity in this study, were assessed in the $A$. aegypti orco-GAL4, Or1-GAL4 and Or8-GAL4 strains. These were compared to the endogenous gene expression patterns of the orco, Or1 and Or8 receptors, which were first reported in larvae by Mysore et al. [26] (shown here for comparison in Fig. 2a, b, d) and were also characterized in the adult antenna (Fig. $3 \mathrm{c}-\mathrm{e}$ ) in the present investigation. In the orco-GAL4 line, reporter expression, like endogenous orco expression in $A$. aegypti (Figs. 2a, 3c) and EGFP expression in the D. melanogaster reporter line (Fig. 1e), is detected in all Aedes antennal ORNs at both the larval (Fig. 2a1) and adult (Fig. 3c1) stages. Although the orco CRE did not promote EGFP expression outside of the $D$. melanogaster antenna, transgene expression was detected in the A. aegypti orco-GAL4 brain (KM, unpublished observation). 


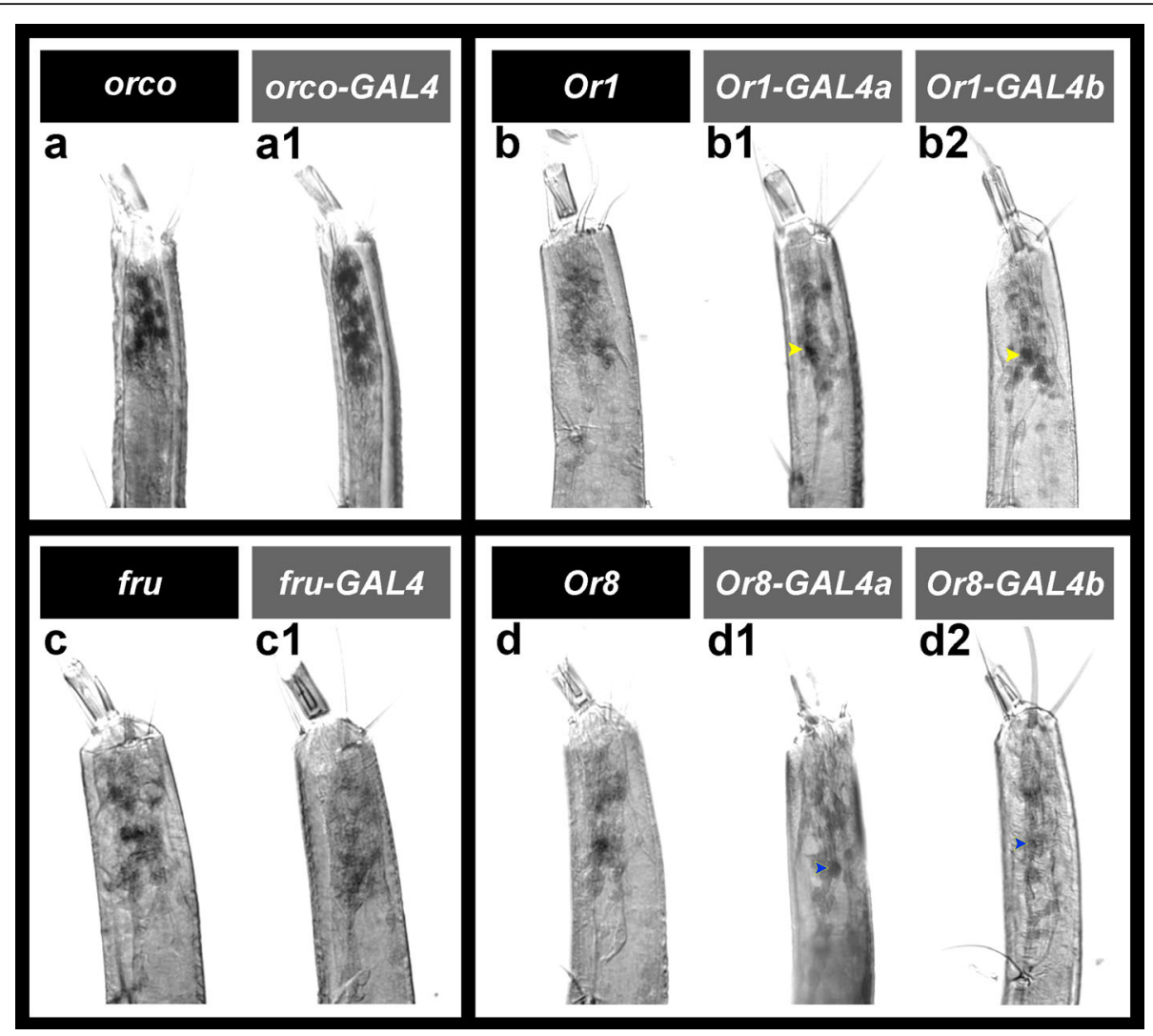

Fig. 2 CRE activity in the A. aegypti larval antenna. FAIRE DNA elements associated with the orco (a1), Or1 (b1, b2), fru (c1) and Or8 (d1, d2) genes promote GAL4 reporter expression in A. aegypti larval ORNs. The orco (a1) CRE promotes transgene expression in all larval ORNs, while the Or1 (b1, b2), fru (c1) and Or8 (d1, d2) CREs drive transgene expression in subsets of ORNs. These expression patterns are comparable to the patterns of native orco (a), Orl (b), fru (c) and Or8 (d) transcripts in the larval antenna. CRE activity is comparable in two separate Or1-GAL4 lines (a in panel b1 and $b$ in panel b2), as well as two separate Or8-GAL4 strains ( $a$ in panel $\mathbf{d} \mathbf{1}$ and $b$ in panel $\mathbf{d} \mathbf{2}$ ). Mean gray value analyses for the cell marked by the yellow arrowheads in $\mathbf{b} \mathbf{1}$ versus $\mathbf{b} \mathbf{2}$ revealed no significant differences in transgene signal intensity levels $(P>0.05)$. Likewise, no differences in transgene signal intensity levels were detected for the cell marked by the blue arrowheads in $\mathbf{d} \mathbf{1}$ versus $\mathbf{d} \mathbf{2}$. Proximal is oriented upward in all panels

Based on the Drosophila reporter assays (Fig. 1f, g), it was predicted that the Or1 and Or8 CREs would promote gene expression in subsets of ORNs. As predicted, the Or1 CRE drove transgene expression in a subset of ORNs in the A. aegypti larval antenna (Fig. 2b1, b2) and the adult $A$. aegypti antenna (Fig. 3d1, d2) of the Or1-GAL4a and $b$ strains. Comparable patterns of transgene expression were observed in the two strains (Fig. $2 \mathrm{~b} 1$ versus b2; Fig. $3 \mathrm{~d} 1$ versus d2), and transgene expression in the two Aedes strains matched that of endogenous Or1 expression (larval expression is shown in Fig. 2b and adult expression in Fig. 3d). Furthermore, mean gray value comparisons of the cells marked in Fig. $2 \mathrm{~b} 1$ versus b2 (t-test: $t_{(24)}=1.27, P=0.22$ ) or Fig. $3 \mathrm{~d} 1$ versus $\mathrm{d} 2$ (t-test: $t_{(14)}=0.30, P=0.77$ ) detected no significant differences in transgene signal intensity levels between the Or1-GAL4a and $b$ strains. Likewise, transgene expression patterns in the Or8-GAL4a and $b$ lines were comparable (Fig. $2 \mathrm{~d} 1$ versus $\mathrm{d} 2$ and Fig. $3 \mathrm{e} 1$ versus $\mathrm{e} 2$ ) and mimicked that of endogenous $\mathrm{Or} 8$ gene expression
(Fig. 2d; 3e) in both the larval (Fig. 2d1, d2) and adult (Fig. 3e1, e2) A. aegypti antenna. Levels of transgene expression were comparable between the Or8-GAL4a and $b$ strains, with no significant differences detected in mean gray values for the cell marked in Fig. $2 \mathrm{~d} 1$ versus d2 (t-test: $\left.t_{(24)}=1.81, P=0.08\right)$ or the cells marked in Fig. 3e1 versus e2 (t-test: $\left.t_{(14)}=0.68, P=0.51\right)$.

\section{Identification of a fru CRE that drives sex-specific gene expression in the A. aegypti antenna}

As discussed above, a FAIRE DNA element associated with the A. aegypti fru gene drove male-specific reporter expression in a subset of Drosophila ORNs (Fig. 1d, i). This $A$. aegypti FAIRE DNA element was found to be active in a subset of larval (Fig. 2c1) and adult male (Fig. 3a1) ORNs of the fru-GAL4 A. aegypti strain. The pattern of transgene expression mimicked that of endogenous fru expression (Fig. 2c; 3a) in the A. aegypti larval (Fig. 2c1) and adult male (Fig. 3a1) antenna; however, transgene 


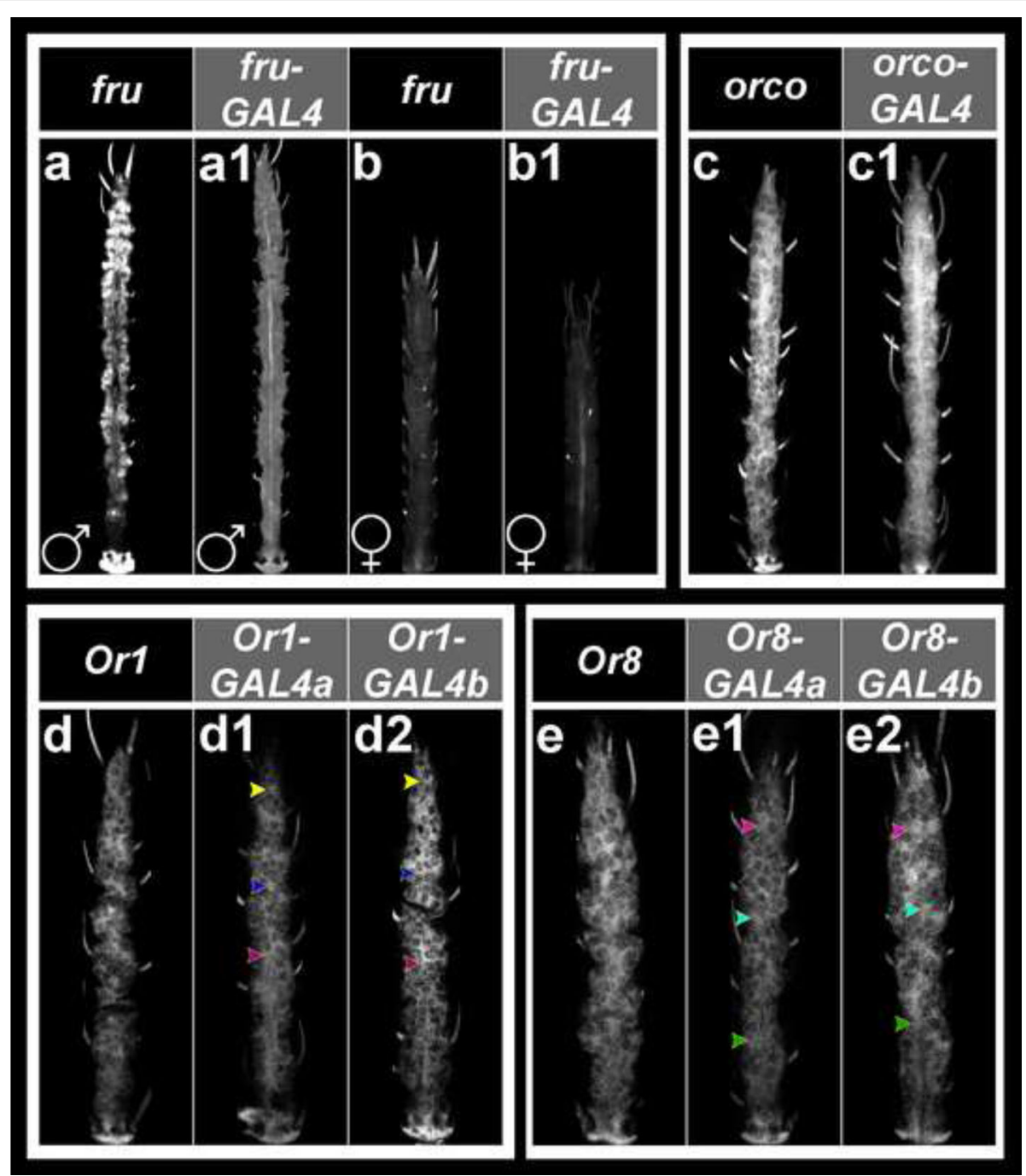

Fig. 3 CRE activity in A. aegypti adult antennal ORNs. Expression of GAL4 transcripts driven by FAIRE DNA elements adjacent to the fru (male in a1), orco (c1), Or1 (d1, d2) and Or8 (e1, e2) genes are comparable to expression of native fru (male in a), orco (c), Or1 (d) and Or8 (e) transcripts in the adult $A$. aegypti antenna. No fru transcript is detected in the A. aegypti female antenna (b), and GAL4 expression is not driven by the fru CRE in the female antenna (b1). CRE activity is comparable in two separate Or8-GAL4 lines ( $a$ in $\mathbf{e} 1$ and $b$ in e2), as well as two separate Or1-GAL4 strains ( $a$ in panel $\mathbf{d} \mathbf{1}$ and $b$ in panel $\mathbf{d} \mathbf{2}$ ). Mean gray value analyses for the cells marked by the yellow, blue, or purple arrowheads in $\mathbf{d} \mathbf{1}$ versus $\mathbf{d} \mathbf{2}$ revealed no significant differences in transgene signal intensity levels $(P>0.05)$. Likewise, no significant differences were detected in the transgene signal intensity levels of the cells marked by the magenta, cyan, or green arrowheads in $\mathbf{e} 1$ versus $\mathbf{e} 2(P>0.05)$. The fru (male in a1), Or1 (d1, d2) and Or8 (e1 and e2) CREs are active in subsets of ORNs, while the orco (c1) CRE promotes gene expression in all ORNs. With the exception of $\mathbf{a}$ and $\mathbf{a} \mathbf{1}$, in which male antennae are shown, female antennae oriented proximal upward are displayed in all panels

expression detected in the fru-GAL4 adult male antenna was somewhat weaker than larval expression (Fig. 2c1) or that of endogenous fru expression (Fig. 3a). Neither endogenous fru expression (Fig. 3b) nor CRE activity (Fig. 3b1) were detected in the adult female antenna. Thus, an $A$. aegypti FAIRE DNA element associated with the fru gene promotes sex-specific gene expression in $A$. aegypti adult male antennal ORNs. It is possible that endogenous larval fru expression and CRE activity in the fruGal4 strains was also sexually dimorphic in larval ORNs, but this was not assessed because $A$. aegypti males and females are not physically distinguishable as larvae.

\section{Discussion}

Identification of $A$. aegypti CREs that function similarly in the ORNs of divergently-related dipteran insects

The results of this investigation indicated that the $A$. aegypti Or1, Or8, orco and fru CREs identified through FAIRE-seq open chromatin profiling [17] can promote transgene expression patterns in the $A$. aegypti antenna that are comparable to the endogenous Or1, Or8, orco 
and fru gene transcript expression patterns in A. aegypti larval (Fig. 2) and adult (Fig. 3) antennae. These findings, in combination with our previous studies [17], provide evidence that FAIRE-seq is a powerful method for identification of CREs in mosquitoes. Given the success of the first study in $A$. aegypti [17], additional FAIRE-seq open chromatin profiling studies would be useful both in $A$. aegypti and other mosquitoes, as well as other non-model organisms. In an effort to identify CREs for multiple different tissue types, whole embryos were selected for our initial proof of concept studies in $A$. aegypti [17]. Analyses of these FAIRE-seq embryonic data (here and in [17]) confirmed that CREs for multiple different tissues were in fact identified, while also demonstrating that some of the CREs identified in embryos can function at later life stages. However, given that nucleosome positioning is influenced by multiple factors and can vary in different tissues and at different life stages (see reference [15] for a useful review), it will undoubtedly be useful to pursue tissue-specific FAIRE-seq open chromatin profiling directly in tissues of interest and at multiple mosquito life stages. For example, while the current investigation successfully identified multiple regulatory elements for the adult antennae, a FAIRE-seq investigation performed directly with tissue prepared from the adult antenna would likely uncover regulatory elements for additional Or genes. Likewise, although a sex-specific regulatory element was fortuitously identified in the present study, the direct comparison of open chromatin profiles in male versus female mosquito tissues of interest, including the antenna, would most likely identify additional CREs that promote sex-specific transcription and reveal insight into epigenetic mechanisms underlying sexually dimorphic traits.

As shown in this investigation, FAIRE-seq can be paired with secondary screening of elements of interest in $D$. melanogaster reporter assays to facilitate detection of CREs that promote gene expression in tissues of interest. The conservation of antennal CRE activity observed between $A$. aegypti and D. melanogaster in this investigation likely results from a conserved transcriptional regulatory code which is believed to regulate $O r$ gene expression in the two species [26, 31]. In most cases, with orco being an exception, mosquito and fruit fly $\mathrm{Or}$ genes are not direct orthologues [27]. However, the functions of transcription factors that regulate $O r$ gene expression in the antenna, including E93, Onecut, and Acj6, do appear to be conserved [26]. It is anticipated that since these $A$. aegypti CREs drive tissue-specific reporter expression in Drosophila that mimics the activity of the elements in A. aegypti, these CREs are likely to function similarly in other dipterans, including additional vector mosquito species that are more closely related to $A$. aegypti than are fruit flies. To test this, it may be interesting to transform other mosquitoes with the transgenic constructs generated in this investigation, which are compatible with $p B a c$ and phiC31-mediated transgenesis in multiple insect species. Finally, it is important to note that although the four antennal CREs characterized in this investigation appear to function comparably in $A$. aegypti and D. melanogaster, this will not be the case for all CREs. It is also important to uncover cases in which CRE function has diverged between the two species, and this will be a subject of future investigations.

Although $A$. aegypti orco is not expressed in the brain, ectopic CRE activity was detected in the A. aegypti orco-GAL4 brain. Ectopic GAL4 expression has been detected in the brains of other strains transformed with this construct ([21], KM and MDS unpublished) and is believed to be dependent on position-dependent variation in transgene insertion. Although the PB-GAL4 $E C F P$ construct used in this investigation is compatible with both phiC31 and piggybac (pBac) transgenesis [21], high-efficiency phiC31 acceptor lines for site-specific integration are not openly available for $A$. aegypti. Use of site-specific integration would eliminate variation in transgene expression that can result from variable integration sites which can result when $p B a c$ transgenesis is utilized. Although several Or1-GAL4, Or8-GAL4 and fru-GAL4 strains which did not contain this ectopic brain expression domain were successfully generated in this investigation, it may nevertheless be useful to construct high-efficiency $A$. aegypti attP strains that would permit site-specific integration of constructs in the future.

\section{Developing a toolkit for manipulation of mosquito neurons}

The $A$. aegypti strains generated in this investigation can significantly enhance neurogenetic analysis of olfactory system function in $A$. aegypti. In this study, CREs were cloned upstream of the GAL4 transgene, which was used as a reporter for characterizing the activity of these CREs, but could also be used to drive expression of transgenes of interest in ORNs in the future. This will require generation of UAS responder lines for manipulation of neural gene expression and neural function in $A$. aegypti. It would be useful to generate UAS responder lines that express dsRNA to block gene function in specific neurons of interest. It would also be interesting to generate UAS responders that can function as neural tracers. For example, the plant lectin wheat germ agglutinin (WGA) has been used to trace neural circuits and mark synaptic activity in genetic model organisms, as it can be transported by axons and dendrites in both anterograde and retrograde directions, and it can be transferred across synapses [32]. GAL4-driven expression of $U A S-W G A$ in the Drosophila nervous system, which can be detected through immunohistochemical staining with 
an antibody against WGA, has been used to uncover visual, motor neuron, and gustatory circuitries in Drosophila [32-34]. UAS constructs that drive targeted neural cell ablation would also be of interest. For example, an attenuated version of diptheria toxin is useful for ablation of specific cells [3] and has been used in neural cell death ablation studies [35]. Furthermore, expression of ion channels, toxins or genetically encoded proteins that activate or silence neural activity has revolutionized the study of neuroscience in genetic model organisms [3]. For example, optogenetics, light triggered neural activation, would be quite useful for analyses of mosquito neural function. Channel rhodopsins, light activated cation channels, the most widely used optogenetic tools for activation of neurons (reviewed by Venken et al. [3]), have yet to be reported in mosquitoes.

\section{Identification of a sex-specific regulatory element in $A$. aegypti}

The FAIRE DNA element flanking the A. aegypti fru gene was found to drive sex-specific gene expression in the $A$. aegypti male antenna which mimicked the activity of this CRE in D. melanogaster reporter assays. These results suggest that some of the mechanisms which regulate sex-specific expression of fru are conserved between the two organisms. Recent studies [10] have demonstrated that the chromatin modulatory protein Alhambra restricts fru expression to specific neurons during development. Signaling from the ORs through CamK and a histone acetyl transferase protein then functions to maintain $f r u$ expression in specific ORNs [10]. It is possible that these mechanisms are conserved in $A$. aegypti. Moreover, in $D$. melanogaster, fru expression acts as a molecular marker to label neural circuits that regulate sex-specific behaviors [36]. If this is also the case in A. aegypti, the results of this investigation have provided both insight into the identity of at least some of the ORNs that are responsible for sexually dimorphic behaviors in $A$. aegypti, as well as a means of modulating gene expression in these neurons for the manipulation of sexually dimorphic behaviors.

In insects, olfaction is a critical component of sexually dimorphic behaviors. Fruit flies sense volatile pheromones and other odors that are critical for courtship behaviors via the olfactory system [37]. In Drosophila, the male splice-form of Fru, Fru ${ }^{\mathrm{M}}$, is necessary and sufficient for regulation of sex-specific behaviors such as aggression and courtship in males [38]. D. melanogaster Fru is expressed in the neural circuit that promotes sexually dimorphic responses to the pheromone cis-vaccenyl acetate, a male-specific pheromone detected by OR67d-positive neurons that suppresses male-male and male-female courtship behavior [36]. Fru is also expressed in the Ir84a-positive class of neurons, which coordinate reproductive behaviors based on the availability of food resources [39]. A third Fru-positive class of ORNs expresses OR47b, which is responsible for detection of methyl laurate, a cuticular pheromone required for successful copulation [40-43]. Fru-positive neurons may behave in a similar fashion to regulate behavioral responses to pheromones and other odors that mediate sex-specific behaviors in $A$. aegypti. It will be interesting to assess which $O r$ genes are expressed by Fru-positive neurons and to functionally characterize the role of Fru in A. aegypti. The fru-GAL4 strain generated in this investigation could prove to be very useful in such investigations and may promote the elucidation of novel strategies for regulation of sexually dimorphic behaviors that contribute to mosquito reproduction or the spread of disease-causing pathogens.

\section{Conclusions}

In conclusion, the results of this investigation demonstrate that FAIRE-seq, when paired with $D$. melanogaster reporter testing of FAIRE DNA elements of interest, is a high-yield method for identification of CREs that may function in mosquito tissues of interest. This methodology could be extended to other insect vectors of disease and other non-model organisms, in which discovery of CREs has been an ongoing challenge. The A. aegypti strains generated and characterized in this investigation expand the genetic toolkit available for the study of Aedes olfaction, including dissection of sensory contributions to sexually dimorphic mosquito behaviors that are critical for reproduction and the spread of disease-causing pathogens. The CREs identified in this investigation drive comparable olfactory neural expression patterns in both A. aegypti and D. melanogaster which likely result from a conserved transcriptional code that regulates $O R$ expression in the two species [26, 31]. It is therefore likely that these CREs, which have been cloned upstream of GAL4 in a transgenic construct that is compatible with transgenesis in multiple insect species, may function similarly in multiple dipteran insects, including other disease vector mosquito species.

\section{Abbreviations \\ acj6: Abnormal chemosensory jump; CRE: Cis-regulatory element; FAIRE: Formaldehyde-assisted isolation of regulatory elements; FAIRE- seq: Formaldehyde-assisted isolation of regulatory elements followed by sequencing; fru: Fruitless; G: Generation; Or: Odorant receptor; Or1: Odorant receptor 1; Or8: Odorant receptor 8; orco: Odorant coreceptor; ORNs: Olfactory receptor neurons; $\mathrm{BBac}$ : Piggybac; WGA: Wheat germ agglutinin}

\section{Acknowledgements}

We thank David O'Brochta, Rob Harrell, Marc Halfon, and Malcolm Frasier for advice on transgenesis. David O'Brochta and Marc Halfon generously provided plasmid constructs for this project. Drosophila transgenic reporter lines were generated by Rainbow Transgenic Flies, Inc. (Camarillo, CA, USA). The Insect Transformation Facility at the University of Maryland College Park generated the mosquito transgenic strains. Thanks to Limb Hapairai and Longhua Sun for assistance with mosquito rearing. 


\section{Funding}

This work was funded by NIH/NIAID Award R21Al117145-01 to MDS. The funding source was not involved in study design, data collection and interpretation, or the decision to submit the work for publication.

\section{Availability of data and materials}

All data generated or analyzed during this study are included in this article.

\section{Authors' contributions}

KM analyzed the Drosophila and Aedes transgenics, characterized CRE activity, performed all of the imaging, prepared all the figures for this manuscript, and assisted with the writing. PL performed all of the cloning in this investigation. MDS prepared the manuscript, conceived of the study, designed experiments, and assisted with data analysis and interpretation. All authors read and approved the final manuscript.

\section{Ethics approval and consent to participate}

Not applicable.

\section{Consent for publication}

Not applicable.

\section{Competing interests}

The authors declare that they have no competing interests.

\section{Publisher's Note}

Springer Nature remains neutral with regard to jurisdictional claims in published maps and institutional affiliations.

\section{Author details}

${ }^{1}$ Department of Medical and Molecular Genetics, Indiana University School of Medicine, 1234 Notre Dame Avenue, Raclin-Carmichael Hall, South Bend, IN 46617, USA. ${ }^{2}$ The University of Notre Dame Eck Institute for Global Health, Notre Dame, IN 46556, USA. ${ }^{3}$ Department of Biological Sciences, The University of Notre Dame, Notre Dame, IN 46556, USA.

\section{Received: 13 March 2018 Accepted: 27 June 2018}

\section{Published online: 11 July 2018}

\section{References}

1. Centers for Disease Control and Prevention. Surveillance and control of Aedes aegypti and Aedes albopictus. http://www.cdc.gov/chikungunya/ resources/vector-control.html (2016). Accessed May 2016.

2. Duman-Scheel M, Syed Z. Developmental neurogenetics of sexual dimorphism in Aedes aegypti. Front Ecol Evol. 2015;3:61.

3. Venken KJ, Simpson JH, Bellen HJ. Genetic manipulation of genes and cells in the nervous system of the fruit fly. Neuron. 2011:72:202-30.

4. Owald D, Lin S, Waddell S. Light, heat, action: neural control of fruit fly behaviour. Philos Trans R Soc Lond B Biol Sci. 2015:370:20140211.

5. Kimura K. Role of cell death in the formation of sexual dimorphism in the Drosophila central nervous system. Dev Growth Differ. 2011:53:236-44.

6. Goodwin SF, Taylor BJ, Villella A, Foss M, Ryner LC, Baker BS, et al. Aberrant splicing and altered spatial expression patterns in fruitless mutants of Drosophila melanogaster. Genetics. 2000;154:725-45.

7. Anand A, Villella A, Ryner LC, Carlo T, Goodwin SF, Song HJ, et al. Molecular genetic dissection of the sex-specific and vital functions of the Drosophila melanogaster sex determination gene fruitless. Genetics. 2001;158:1569-95.

8. Manoli DS, Foss M, Villella A, Taylor BJ, Hall JC, Baker BS. Male-specific fruitless specifies the neural substrates of Drosophila courtship behaviour. Nature. 2005:436:395-400.

9. Demir E, Dickson BJ. fruitless splicing specifies male courtship behavior in Drosophila. Cell. 2005;121:785-94.

10. Hueston CE, Olsen D, Li Q, Okuwa S, Peng B, Wu J, et al. Chromatin modulatory proteins and olfactory receptor signaling in the refinement and maintenance of fruitless expression in olfactory receptor neurons. PLoS Biol. 2016;14:e1002443.

11. Salvemini M, D'Amato R, Petrella V, Aceto S, Nimmo D, Neira M, et al. The orthologue of the fruitfly sex behaviour gene fruitless in the mosquito Aedes aegypti: evolution of genomic organisation and alternative splicing. PLoS One. 2013;8:e48554.
12. Kokoza VA, Raikhel AS. Targeted gene expression in the transgenic Aedes aegypti using the binary Gal4-UAS system. Insect Biochem Mol Biol. 2011;41:637-44.

13. Simon JM, Giresi PG, Davis IJ, Lieb JD. Using formaldehyde-assisted isolation of regulatory elements (FAIRE) to isolate active regulatory DNA. Nat Protoc. 2012;7:256-67.

14. Giresi PG, Kim J, McDaniell RM, lyer VR, Lieb JD. FAIRE (FormaldehydeAssisted Isolation of Regulatory Elements) isolates active regulatory elements from human chromatin. Genome Res. 2007:17:877-85.

15. Henikoff S. Nucleosome destabilization in the epigenetic regulation of gene expression. Nat Rev Genet. 2008;9:15-26.

16. Giresi PG, Lieb JD. Isolation of active regulatory elements from eukaryotic chromatin using FAIRE (Formaldehyde Assisted Isolation of Regulatory Elements). Methods. 2009:48:233-9.

17. Behura SK, Sarro J, Li P, Mysore K, Severson DW, Emrich SJ, et al. Highthroughput cis-regulatory element discovery in the vector mosquito Aedes aegypti. BMC Genomics. 2016;17:341.

18. Clemons A, Mori A, Haugen M, Severson DW, Duman-Scheel M. Culturing and egg collection of Aedes aegypti. Cold Spring Harb Protoc. 2010;10:pdb.prot5507.

19. Flybase. Bloomington Drosophila Stock Center. 2015. http://flybase.org/ reports/FBst0009744. Accessed March 2015.

20. Patel NH. Imaging neuronal subsets and other cell types in whole-mount Drosophila embryos and larvae using antibody probes. Methods Cell Biol. 1994:44:445-87.

21. O'Brochta DA, Pilitt KL, Harrell RA 2nd, Aluvihare C, Alford RT. Gal4-based enhancer-trapping in the malaria mosquito Anopheles stephensi. G3 (Bethesda). 2012;2:1305-15.

22. Berghammer AJ, Klingler M, Wimmer EA. A universal marker for transgenic insects. Nature. 1999:402:370-1.

23. Clemons A, Haugen M, Flannery E, Kast K, Jacowski C, Severson D, et al. Fixation and preparation of developing tissues from Aedes aegypti. Cold Spring Harb Protoc. 2010;10:pdb.prot5508.

24. Haugen M, Tomchaney M, Kast K, Flannery E, Clemons A, Jacowski C, et al. Whole-mount in situ hybridization for analysis of gene expression during Aedes aegypti development. Cold Spring Harb Protoc. 2010;10:pdb.prot5509.

25. Patel NH. In situ hybridization to whole mount Drosophila embryos. In: Krieg PA, editor. A laboratory guide to RNA: Isolation, analysis, and synthesis. New York: Wiley-Liss; 1996. p. 357-70.

26. Mysore K, Andrews E, Li P, Duman-Scheel M. Chitosan/siRNA nanoparticle targeting demonstrates a requirement for single-minded during larval and pupal olfactory system development of the vector mosquito Aedes aegypti. BMC Dev Biol. 2014;14:9.

27. Bohbot J, Pitts RJ, Kwon HW, Rutzler M, Robertson HM, Zwiebel LJ. Molecular characterization of the Aedes aegypti odorant receptor gene family. Insect Mol Biol. 2007:16:525-37.

28. DeGennaro M, McBride CS, Seeholzer L, Nakagawa T, Dennis EJ, Goldman C, et al. orco mutant mosquitoes lose strong preference for humans and are not repelled by volatile DEET. Nature. 2013;498:487-91.

29. Bohbot JD, Dickens JC. Characterization of an enantioselective odorant receptor in the yellow fever mosquito Aedes aegypti. PLoS One. 2009;4:e7032.

30. Yamamoto D, Koganezawa M. Genes and circuits of courtship behaviour in Drosophila males. Nat Rev Neurosci. 2013;14:681-92.

31. Jafari S, Alkhori L, Schleiffer A, Brochtrup A, Hummel T, Alenius M. Combinatorial activation and repression by seven transcription factors specify Drosophila odorant receptor expression. PLoS Biol. 2012;10:e1001280.

32. Yoshihara $Y$, Mizuno T, Nakahira M, Kawasaki M, Watanabe $Y$, Kagamiyama $H$, et al. A genetic approach to visualization of multisynaptic neural pathways using plant lectin transgene. Neuron. 1999;22:33-41.

33. Tabuchi K, Sawamoto K, Suzuki E, Ozaki K, Sone M, Hama C, et al. GAL4/ UAS-WGA system as a powerful tool for tracing Drosophila transsynaptic neural pathways. J Neurosci Res. 2000:59:94-9.

34. Kunzle H, Radtke-Schuller S. Oligosynaptic pathways possibly relaying visceral and/or gustatory information to the olfactory bulb in the hedgehog tenrec. Neurosci Lett. 2001:303:53-6.

35. Berdnik D, Chihara T, Couto A, Luo L. Wiring stability of the adult Drosophila olfactory circuit after lesion. J Neurosci. 2006;26:3367-76.

36. Kurtovic A, Widmer A, Dickson BJ. A single class of olfactory neurons mediates behavioural responses to a Drosophila sex pheromone. Nature. 2007:446:542-6.

37. Rodrigues $\mathrm{V}$, Hummel T. Development of the Drosophila olfactory system. Adv Exp Med Biol. 2008;628:82-101. 
38. Stockinger P, Kvitsiani D, Rotkopf S, Tirian L, Dickson BJ. Neural circuitry that governs Drosophila male courtship behavior. Cell. 2005;121:795-807.

39. Grosjean Y, Rytz R, Farine JP, Abuin L, Cortot J, Jefferis GS, et al. An olfactory receptor for food-derived odours promotes male courtship in Drosophila. Nature. 2011;478:236-40.

40. Root CM, Masuyama K, Green DS, Enell LE, Nassel DR, Lee CH, et al. A presynaptic gain control mechanism fine-tunes olfactory behavior. Neuron. 2008:59:311-21.

41. Wang L, Han X, Mehren J, Hiroi M, Billeter JC, Miyamoto T, et al. Hierarchical chemosensory regulation of male-male social interactions in Drosophila. Nat Neurosci. 2011;14:757-62.

42. Sakurai A, Koganezawa M, Yasunaga K, Emoto K, Yamamoto D. Select interneuron clusters determine female sexual receptivity in Drosophila. Nat Commun. 2013;4:1825

43. Dweck HK, Ebrahim SA, Thoma M, Mohamed AA, Keesey IW, Trona F, et al. Pheromones mediating copulation and attraction in Drosophila. Proc Natl Acad Sci USA. 2015;112:E2829-35.

Ready to submit your research? Choose BMC and benefit from:

- fast, convenient online submission

- thorough peer review by experienced researchers in your field

- rapid publication on acceptance

- support for research data, including large and complex data types

- gold Open Access which fosters wider collaboration and increased citations

- maximum visibility for your research: over $100 \mathrm{M}$ website views per year 